\title{
PRODUCTS OF UNCOUNTABLY MANY $k$-SPACES
}

\author{
N. NOBLE
}

\begin{abstract}
It is shown that if a product of nonempty spaces is a $k$-space then for each infinite cardinal $n$ some product of all but $n$ of the factors has each $n$-fold subproduct $n-\kappa_{0}$-compact (each $n$-fold open cover has a finite subcover). An example is given, for each regular $\mathfrak{n}$, of a space $X$ which is not $n-\boldsymbol{\kappa}_{0}$-compact (so $X^{\mathfrak{n}^{+}}$is not a $k$-space) for which $X^{\mathfrak{n}}$ is a $k$-space.
\end{abstract}

1. Introduction. A subset $F$ of a topological space $X$ is $k$-closed if $F \cap K$ is closed in $K$ for each compact subset $K$ of $X$. A space in which each $k$-closed subset is closed is called a $k$-space. (No separation axioms will be assumed, so this definition differs from some of the other published definitions.) Although conditions under which finite or countable products of $k$-spaces will be $k$-spaces have been extensively studied, for instance in [1], [2], [4], [6], and [7], the only noteworthy results concerning products of $k$-spaces having uncountably many factors are included in the fact, proved in [5], that for a product of nonempty $T_{1}$-spaces to be a $k$-space, some product of all but countably many of its factors must be countably compact. We improve and extend this result with:

THEOREM. If a product of nonempty spaces is a $k$-space then, for each infinite cardinal $\mathfrak{n}$, some product of all but $\mathfrak{n}$ of its factors has each $\mathfrak{n}$-fold subproduct $\mathrm{n}-\aleph_{0}$-compact.

Recall that a space is $n-\aleph_{0}$-compact if each $n$-fold open cover contains a finite subcover. As an immediate consequence of this theorem (together with Tychonoff's Theorem) we have:

COROLlary. All powers of a space $X$ are $k$-spaces if and only if $X$ is compact.

It is amusing to contrast this result with the fact, established in [8], that all powers of a $T_{1}$-space $X$ are normal if and only if $X$ is compact. (Thus all powers of a $T_{1}$-space $X$ are $k$-spaces if and only if all powers of

Received by the editors December 2, 1970.

AMS 1969 subject classifications. Primary 5425, 5452.

Key words and phrases. Product spaces, $k$-spaces, spaces with weak topologies.

(C) American Mathematical Society 1972 
$X$ are normal.) The strength of our theorem on $k$-spaces is indicated by the following:

EXAMPLE. For each regular cardinal $\mathfrak{n}$ there exists a space $X$ such that $X^{\mathfrak{n}}$ is a $k$-space but $X^{\mathfrak{m}}$ is not a $k$-space for any larger cardinal $\mathrm{m}$.

Indeed, $X$ can be taken to be $n$. (As usual, a cardinal $n$ is identified with the smallest ordinal of cardinality $\mathfrak{n}$ and, unless otherwise indicated, is assumed to bear the order topology.) This space $X$ is certainly not $\mathrm{n}-\boldsymbol{\kappa}_{0}$-compact so, by the Theorem, $X^{\mathrm{m}}$ is not a $k$-space for any $\mathrm{m}$ greater than $\mathrm{n}$. That $X^{\mathfrak{n}}$ is a $k$-space will follow from the more general considerations below.

Call a space n-determined if a subset is closed whenever it meets each subset $S$ having n or fewer elements in a set which is closed in $S$. Recall that a space is $n$-bounded if each subset with $n$ or fewer elements is contained in a compact set. Clearly $n$-boundedness is preserved by arbitrary products and each $\mathrm{n}$-bounded $\mathrm{n}$-determined space is a $k$-space.

Proposition 1. For $\mathfrak{n}$ an infinite cardinal, an $\mathrm{m}$-fold product of $\mathrm{n}$ determined spaces is $\mathrm{n}$-determined if and only if all but at most $\mathrm{n}$ of the factors are indiscrete.

We call a space $<n$-bounded if each subset with fewer than $n$ elements is contained in a compact set and we call a space $<n$-determined if a subset is closed whenever it meets each subset $S$ having fewer than $\mathfrak{n}$ elements in a set which is closed in $S$. Note that if $X=\mathfrak{n}$ and $n$ is regular, then $X$ is $<\mathrm{n}$-bounded and $<\mathrm{n}$-determined. Thus our next result shows that, for this $X, X^{\mathfrak{n}}$ is a $k$-space.

Proposition 2. Let $X=\prod_{\alpha \in \mathfrak{n}} X_{\alpha}$. If each $X_{\alpha}$ is $<\mathrm{n}$-bounded and $<\mathrm{n}$ determined, then $X$ is a $k$-space.

\section{Proofs.}

Proof of THE THeORem. The proof is by induction on $\mathfrak{n}$, so suppose that the Theorem holds for each cardinal less than $n$ and that $X=\prod_{\alpha \in \mathfrak{n}^{+}} X_{\alpha}$ is a nonempty $k$-space. In order to show that some product of all but at most $n$ of the factors of $X$ has each $n$-fold subproduct $n-\aleph_{0}$-compact it suffices, by [5, Theorem 1], to show that all but $n$ of them must be $\mathrm{n}-\boldsymbol{\aleph}_{0}$-compact. Suppose that this is not the case; since by the induction hypothesis all but at most $m$ of the factors are $\mathfrak{m}-\boldsymbol{\aleph}_{0}$-compact for each $m$ less than $\mathfrak{n}$, we may suppose that each $X_{\alpha}$ has an $\mathfrak{n}$-fold open cover which has no subcover of smaller cardinality. Passing to complements of unions, each $X_{\alpha}$ thus contains a nested family $\left\{A_{\alpha}^{\lambda}: \lambda \in \mathfrak{n}\right\}$ of nonempty closed sets with $\bigcap\left\{A_{\alpha}^{\lambda}: \lambda \in \mathfrak{n}\right\}=\varnothing$. Further, we may suppose that for each $\alpha$ there exists a point $y_{\alpha}$ in $X \backslash A_{\alpha}^{0}$.

For each $\lambda$ in $n$ let $B_{\lambda}$ be the union, over all $\gamma$ in $\mathrm{n}^{+}$, of the product sets 
whose $\alpha$ th factor is $\left\{y_{\alpha}\right\}$ for $\gamma \leqq \alpha \leqq \gamma+\lambda$ and $A_{\alpha}^{\lambda}$ otherwise. Let $C_{\lambda}$ be the closure of $\bigcup_{\beta \leqq \lambda} B_{\beta}$ and set $C=\bigcup_{\lambda \in \mathfrak{n}} C_{\lambda}$; we will show that $C$ is $k$-closed but not closed.

To see that $C$ is not closed, note that since any finite subset of $\mathrm{n}^{+}$is contained in a segment $[\gamma, \gamma+\lambda]$ for some $\gamma$ and $\lambda$, the point $y=\left(y_{\alpha}\right)$ is in the closure of $C$. On the other hand, $y$ is not in $C$ since, for $\lambda$ in $\mathfrak{n}$, $\left(X_{0} \mid A_{0}^{\lambda}\right) \times\left(X_{\lambda+1} \mid A_{\lambda+1}^{\lambda}\right) \times \prod_{\alpha \neq 0 ; \alpha \neq \lambda+1} X_{\alpha}$ is a neighborhood of $y$ which does not meet $\bigcup_{\beta \leqq \lambda} B_{\beta}$, so $y$ is not in the closure of $C_{\lambda}$. Now let $K \subseteq \prod_{\alpha} X_{\alpha}$ be compact, say $K=\prod_{\alpha} K_{\alpha}$. We show that $K \cap C$ is closed by showing $K \cap$ $C=K \cap C_{\lambda}$ for some $\lambda$-since $C_{\lambda}$ is closed, this suffices. For each $\alpha$, note that $K_{\alpha}$ cannot meet cofinally many of the decreasing family $\left\{A_{\alpha}^{\lambda}: \lambda \in \mathfrak{n}\right\}$ since its intersection is empty. Thus there exists a $\lambda(\alpha)$ in $n$ such that $K_{\alpha} \cap A_{\alpha}^{\lambda}=$ $\varnothing$ for each $\lambda>\lambda(\alpha)$. Since the domain of $\lambda$ is $\mathfrak{n}^{+}$while its range is $\mathfrak{n}$, there exists a $\lambda_{0}$ in $\mathfrak{n}$ such that $\left\{\alpha: \lambda(\alpha)=\lambda_{0}\right\}$ has cardinality $\mathfrak{n}^{+}$. For $\lambda>$ $\lambda_{0} K \cap C_{\lambda}=K \cap C_{\lambda_{0}}$ since for each point $x$ in the closure of $\bigcup\left\{B_{\beta}: \lambda_{0}<\beta \leqq \lambda\right\}$, $x_{\alpha}$ is in $A_{\alpha}^{\lambda^{2}+1}$ with fewer than $n$ exceptions. Consequently $K \cap C=K \cap C_{\lambda_{0}}$, so $K \cap C$ is closed. This contradicts the hypothesis that $\prod_{\alpha} X_{\alpha}$ is a $k$-space and thus completes the proof.

The proof above is a generalization of the proof sketched in [3, Exercise 7-J]. The first observation of our next proof implies that each subspace of an $n$-determined space is $n$-determined.

Proof of Proposition 1. Let us first note that if $X$ is n-determined and $x$ is in the closure of a subset $A$ of $X$, then $x$ is in the closure of some $\mathrm{n}$-fold or smaller subset of $A$ : Since an $\mathrm{n}$-fold union of sets of cardinality $\mathrm{n}$ itself has cardinality $\mathrm{n}$, the operator which adjoins to $A$ the closures of all of its n-fold subsets is idempotent, and is therefore the closure operator. Now suppose that $X=\prod_{\alpha \in \mathfrak{n}} X_{\alpha}$ where each $X_{\alpha}$ is n-determined and let $x$ be in the closure of a subset $A$ of $X$. We will show that $X$ is n-determined by showing that $x$ is in the closure of some n-fold subset of $A$.

Let $F$ be any finite subset of $n$. Since $x$ is in the closure of $A, \pi_{F}(x)$ is in the closure of $\pi_{F}(A)$, and hence, for some $\mathrm{n}$-fold or smaller subset $A_{F}$ of $A, \pi_{F}(x)$ is in the closure of $\pi_{F}\left(A_{F}\right)$. Let $A^{\prime}=\bigcup\left\{A_{F}: F \subseteq \mathfrak{n}\right\}$ is finite and note that the cardinality of $A^{\prime}$ is less than or equal to $\mathrm{n}$. Since $x$ is clearly in the closure of $A^{\prime}, A^{\prime}$ is as desired.

For the converse, suppose $X=\prod_{\alpha \in \mathfrak{n}^{+}} X_{\alpha}$ where each $X_{\alpha}$ contains a point $x_{\alpha}$ and a closed subset $F_{\alpha}$ with $x_{\alpha}$ not in $F_{\alpha}$. Let $x$ be the point $\left(x_{\alpha}\right)$ and let $F$ be the set of points in $X$ whose $\alpha$ th coordinates, with at most $\mathfrak{n}$ exceptions, lie in $F_{\alpha}$. Clearly $F$ meets each $n$-fold or smaller set in a closed set. Since $x$ is in the closure of $F$ but is not in $F, F$ is not closed, so this shows that $X$ is not n-determined.

Proof of Proposition 2. Let $A \subseteq X$ be $k$-closed and let $x$ be any point in the closure of $A$. We will produce a subset $A^{\prime}$ of such that $x$ is in the 
closure of $A^{\prime}$ and such that, for each $\alpha$ in $\mathfrak{n}, \pi_{\alpha} A^{\prime}$ has cardinality less than n. Since each $X_{\alpha}$ is $<\mathrm{n}$-bounded, each $\pi_{\alpha} A^{\prime}$, and hence $A^{\prime}$ itself, is contained in a compact set. It follows that $x$ must be in $A$ and hence that $X$ is a $k$-space, as desired.

Let $\pi^{\alpha}$ denote the projection from $X$ to $X^{\alpha}=\prod_{\beta<\alpha} X_{\beta}$ and note that, since $\mathfrak{n}$ is regular, the proof of Proposition 1 adapts easily to show that $X^{\alpha}$ is $<n$-determined. We first show that, for each $\alpha, \pi^{\alpha}(x)$ is in $\pi^{\alpha}(A)$. Certainly $\pi^{\alpha}(x)$ is in the closure of $\pi^{\alpha}(A)$ and hence, since $X^{\alpha}$ is $<\mathrm{n}$ determined, $\pi^{\alpha}(x)$ is in the closure of $\pi^{\alpha}(B)$ for some subset $B$ of $A$ having fewer than $\mathrm{n}$ elements. Since $X$ is $<\mathrm{n}$-bounded, $B$ is contained in some compact set $K$. Let $K_{1}$ be the projection of $K$ onto $\prod_{\beta \geq \alpha} X_{\beta}$, and let $K_{2}=\pi^{\alpha}(K) \cup\left\{\pi^{\alpha}(x)\right\}$. Since $A$ is $k$-closed, $A \cap K_{1} \times K_{2}$ is closed in $K_{1} \times K_{2}$ and therefore its projection onto $K_{2}$, which is just $\pi^{\alpha}(A) \cap K_{2}$, is closed in $K_{2}$. Since $\pi^{\alpha}(B) \subseteq \pi^{\alpha}(A) \cap K_{2}$ and $\pi^{\alpha}(x)$ is in the intersection of the closure of $\pi^{\alpha}(B)$ with $K_{2}$, it follows that $\pi^{\alpha}(x)$ is in $\pi^{\alpha}(A)$, as desired.

To construct the set $A^{\prime}$, choose, for each $\alpha$, a point $x^{\alpha}$ in $A$ such that $\pi^{\alpha}\left(x^{\alpha}\right)=\pi^{\alpha}(x)$ and let $A^{\prime}=\left\{x^{\alpha}: \alpha \in \mathfrak{n}\right\}$. It is clear that $A^{\prime}$ has the desired properties, so the proof is complete.

\section{REFERENCES}

1. A. V. Arhangel'skiī, Bicompact sets and topological spaces, Trudy Moskov. Mat. Obšč. 13 (1965), 3-55=Trans. Moscow Math. Soc. 1965, 1-62. MR 33 \#3251.

2. D. E. Cohen, Spaces with weak topology, Quart. J. Math. Oxford Ser. (2) 5 (1954), 77-80. MR 16, 62.

3. J. L. Kelley, General topology, Van Nostrand, Princeton, N.J., 1955. MR 16, 1136.

4. E. Michael, Local compactness and Cartesian products of quotient maps and $k$ spaces, Ann. Inst. Fourier (Grenoble) 18 (1968), fasc. 2, 281-286 MR 39 \#6256.

5. N. Noble, A generalization of a theorem of A. H. Stone, Arch. Math. 18 (1967), 394-395. MR 36 \#5883.

6. - Products of quotient maps and spaces with weak topologies (to appear).

7. - The continuity of functions on Cartesian products, Trans. Amer. Math. Soc. 149 (1970), 187-198. MR 41 \#2636.

8. ——, Products with closed projections. II, Trans. Amer. Math. Soc. 160 (1971), 169-183.

Canary Road, Westlake, Oregon 97493 